\title{
Surgical Thrombectomy of dual coronary artery thrombosis
}

\author{
Mouhammad Kanj ${ }^{1}$, Dany Chawbah ${ }^{2}$, Anis Nassar ${ }^{1}$, and Ziad Mansour ${ }^{1}$ \\ ${ }^{1}$ Lebanese Geitaoui University Medical Centre \\ ${ }^{2}$ Monla Hospital
}

March 17, 2021

\begin{abstract}
Concomitant thrombosis of two coronary arteries is an uncommon condition that is reported to cause sudden cardiac death. In this paper, we describe the case of a 42-year-old man who presented with simultaneous LAD and RCA occlusion causing anterior and inferior myocardial infarctions. PCI was attempted and failed, an Intra-Aortic Balloon Pump (IABP) was inserted and the patient was referred to surgery. Following surgical thrombectomy and coronary artery bypass grafting (CABG), the patient recovered with no complications.
\end{abstract}

\section{Introduction}

Acute myocardial infarction due to simultaneous occlusion of two epicardial vessels has a dismal prognosis with high mortality [1]. Therefore, immediate management is necessary to prevent sudden cardiac death. We present a surgical solution to this critical situation when it cannot be addressed by acute percutaneous therapy.

\section{Case report:}

This report was approved by the institutional review board of the Lebanese Geitaoui University Medical Centre.

The patient signed informed consent for reporting his case.

A 42-year-old male smoker, who hadn't sought medical care before, presented to the emergency department with a two-day-history of persistent chest pain. Electrocardiogram (ECG) showed ST-elevation in precordial leads along with negative T-wave in inferior leads. Troponin-T level was high $(1.92 \mathrm{ng} / \mathrm{ml})$. He had tachycardia (130 beats/min) and low blood pressure $(90 / 50 \mathrm{mmHg})$. Coronary angiography showed total occlusion of both the right coronary artery (RCA) and the left anterior descending artery (LAD) (figure 1); no signs of arterial dissection were found. An Intra-Aortic Balloon Pump (IABP) was inserted to stabilize the patient's hemodynamic status and PCI was attempted but failed. Cardiac ultrasound revealed apical, septal and inferior left ventricular (LV) wall akinesia with a left ventricular ejection fraction (LVEF) of $30 \%$, and no associated valvular disease. The patient was referred to us for surgical treatment. He was admitted to the operating room in a stable hemodynamic status under IABP. CABG was performed under cardio-pulmonary bypass and aortic cross-clamping. During the procedure, both mammary arteries were harvested (both were pedicled to their corresponding subclavian arteries). The RCA contained an organized clot adherent to the vascular wall; it was kept and the arteriotomy closed. The posterior descending artery (PDA) contained fresh clots. These were thrombectomized, then an end-to-side anastomosis with the RIMA was done. The RIMA, still pedicled to its origin, had enough length after adequate skeletonization. The LAD was found to be large $(2.5 \mathrm{~mm}$ in diameter $)$. The LAD-II arteriotomy uncovered a fresh clot that was thrombectomized. The proximal clot was inaccessible through this mid-segment arteriotomy; another arteriotomy was done at the LAD-I level (video 1), and the whole clot was removed. We anastomosed LIMA to LAD-I, side-to-side, then LIMA to LAD-II, end-to-side. Weaning of the cardio-pulmonary bypass, needed 
high doses of intravenous inotropes in addition to the active intra-aortic balloon pump (IABP). The patient was transferred to the cardiac surgery unit in a stable condition with adequate urine output. On the first post-operative day (POD), inotropes started progressive titration and the patient remained stable. The IABP was removed on POD4. The patient became fully autonomous on POD12 and was discharged home. One month later, echocardiography showed antero-apical left ventricular hypokinesia with an improvement of the LVEF reaching 55\%. Haematological workup including factor V-Leiden, protein C, protein S, fibrinogen, anti-thrombin III, Factor Von-Willebrand, and a cardiovascular panel for mutations was completely normal. A follow-up coronary scan (figure 2) performed ten months after surgery showed complete occlusion of RCA, patent RIMA to PDA graft, patent native LAD bed, and patent LIMA to LAD graft.

\section{Discussion:}

Myocardial Infarction due to simultaneous double vessel thromboses is a rarely reported event [1], probably representing $2.5 \%$ among primary percutaneous coronary interventions [2]. Nearly one-third presents with cardiogenic shock, and about one-fourth requires IABP insertion [2]. Contributing factors include inflammatory states, hemodynamic instability, hypercoagulable conditions, prolonged coronary vasospasm, coronary embolism, and hyperhomocysteinemia[2]. However, in more than half of patients, no leading cause can be identified [2]. In our case, no prothrombotic factor was found, and probably heavy tobacco use in such a relatively young age was highly thrombogenic.

An immediate PCI was attempted as recommended by the ACCF/AHA and ESC guidelines, irrespective of the time delay from symptoms onset; when PCI has failed, emergency CABG was indicated and done according to the same recommendations(Class I, Level B) [3, 4]. Routine use of thrombo-aspiration is still not endorsed by the ESC [4]. Our patient was young enough to withstand an extensive surgery, and this proved beneficial. Blood clots were adequately removed from the LAD and the PDA without any crush effect, micro-embolization, or no-reflow phenomenon that may accompany percutaneous therapy. In addition, revascularization using both mammary arteries was successful. This is proven to have the best long-term survival rate [5]. The patient's LAD and LIMA graft were patent on coronary scanner 10 months after the operation despite a theoretical competitive flow. The absence of flow through the completey-occluded-RCA allowed unimpeded flow through RIMA, which proved to be long enough to reach the PDA, and remained patent ten months after surgery. As far as we know, no such reported case was treated surgically; we report a successful surgical management with optimal outcome ten months after surgery.

\section{Conclusion:}

This case proves that surgical thrombectomy and revascularization may be an efficient approach to patients with acute double coronary artery thrombosis with hemodynamic instability when PCI fails, despite the high operative risk.

\section{Acknowledgements:}

We acknowledge Dr Hassan Kanso for the precious contribution in the imaging and interpretation of the coronary scanner, and Dr Mohammad Jomaa for the precious contribution in manuscript reviewing.

\section{Author contributions:}

Mouhammad Kanj: Concept/design, Data analysis/interpretation, Drafting article, Critical revision of article, Data collection.

Dany Chawbah: Concept/design, Data analysis/interpretation, Data collection.

Anis Nassar: Data analysis/interpretation, Data collection.

Ziad Mansour: Concept/design, Data analysis/interpretation, Drafting article, Critical revision of article, Approval of article, Data collection.

\section{References:}


[1] Sia S-K, Huang C-N, Ueng K-C, Wu Y-L, Chan K-C. Double Vessel Acute Myocardial Infarction Showing Simultaneous Total Occlusion of Left Anterior Descending Artery and Right Coronary Artery. Circ J 2008;72:1034-6. doi:10.1253/circj.72.1034.

[2] Pollak PM, Parikh S V., Kizilgul M, Keeley EC. Multiple Culprit Arteries in Patients With ST Segment Elevation Myocardial Infarction Referred for Primary Percutaneous Coronary Intervention. Am J Cardiol 2009;104:619-23. doi:10.1016/j.amjcard.2009.04.053.

[3] 2013 ACCF/AHA Guideline for the Management of ST-Elevation Myocardial Infarction: Executive Summary: A Report of the American College of Cardiology Foundation/American Heart Association Task Force on Practice Guidelines. Catheter Cardiovasc Interv 2013;82:E1-27. doi:10.1002/ccd.24776.

[4] Neumann F-J, Sousa-Uva M, Ahlsson A, Alfonso F, Banning AP, Benedetto U, et al. 2018 ESC/EACTS Guidelines on myocardial revascularization. Eur Heart J 2019;40:87-165. doi:10.1093/eurheartj/ehy394.

[5] Grau JB, Ferrari G, Mak AWC, Shaw RE, Brizzio ME, Mindich BP, et al. Propensity matched analysis of bilateral internal mammary artery versus single left internal mammary artery grafting at 17-year follow-up: validation of a contemporary surgical experience. Eur J Cardio-Thoracic Surg 2012;41:770-6. doi:10.1093/ejcts/ezr213.

\section{Legends:}

Figure 1: (A) EKG upon admission showing ST-elevation in the precordial leads with negative T-wave in the inferior leads. (B) Coronary angiography showing total occlusion of the proximal RCA (white arrow) and (C) proximal LAD (black arrow).

Figure 2: (A) Coronary angioscanner showing (A) LIMA graft and LAD, and (B) a 3D view showing the right subclavian artery (I), RIMA graft (II), the occluded RCA (III) and the patent PDA (IV).

Video 1: Surgical thrombectomy technique.

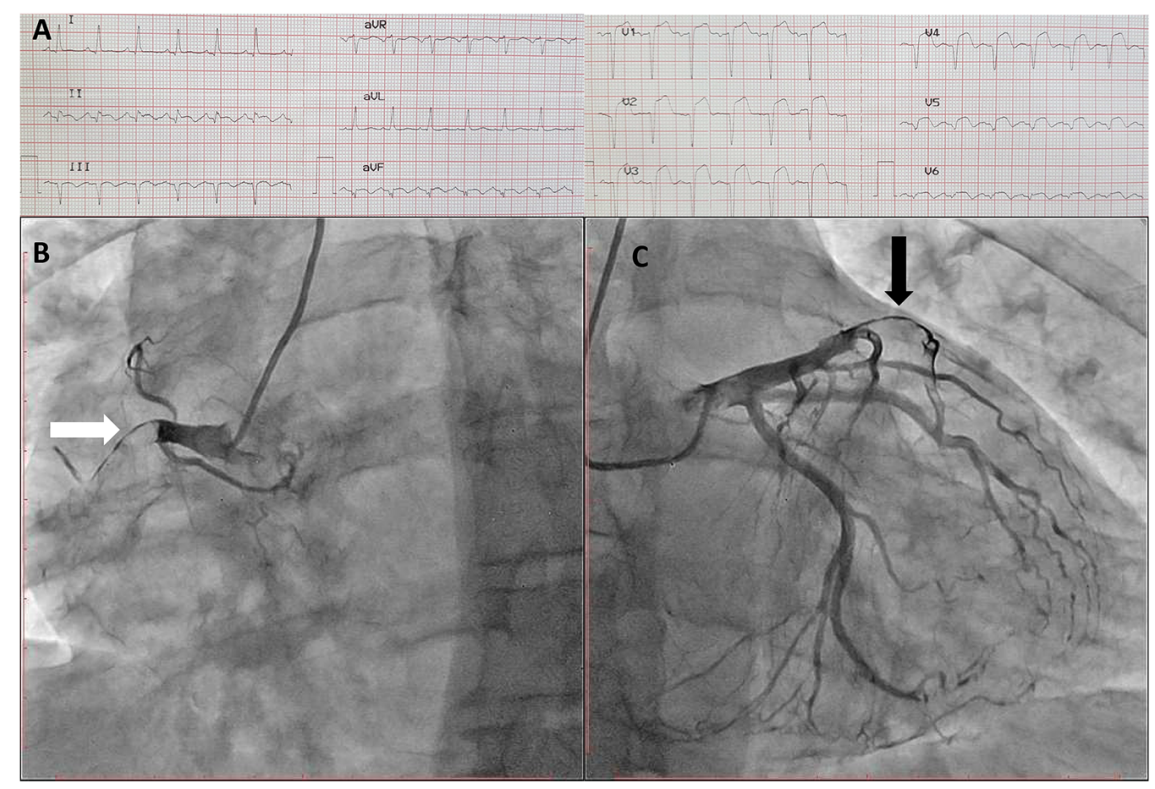




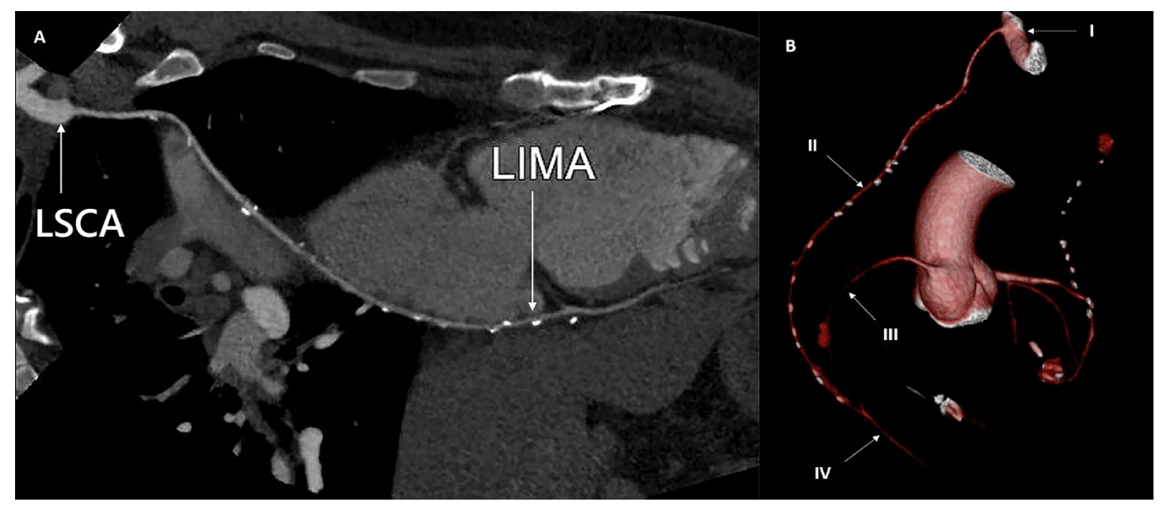

